\title{
A comparative study of ultrasonic c-scan, micro-CT, infrared thermography and Terahertz NDT based on experiments and simulations of composites
}

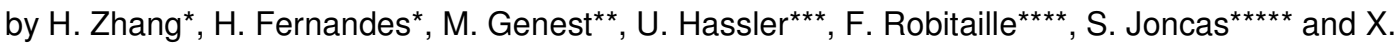 \\ Maldague* \\ * Department of Electrical and Computer Engineering, Computer Vision and Systems Laboratory, Laval \\ University, 1065 Av. de la Medecine, Quebec City, G1V 0A6, Canada \\ **Aerospace Portfolio, Structures, Materials and Manufacturing, National Research Council Canada, 1200 \\ Montreal Road, Ottawa, K1A 0R6, Canada \\ ${ }^{* * *}$ Fraunhofer Development Center X-ray Technologies (EZRT), Department of Application Specific Methods \\ and Systems (AMS), Fraunhofer IIS, Flugplatzstrasse 75, 90768 Fuerth, Germany \\ **** Department of Mechanical Engineering, University of Ottawa, 161 Louis Pasteur, Ottawa, K1N 6N5, Canada \\ ${ }^{* * * * *}$ Department of Automated Manufacturing Engineering, Ecole de Technologie Superieure, 1100 rue Notre- \\ Dame Ouest, Montreal, H3C 1K3, Canada
}

\begin{abstract}
Infrared thermography (IRT) is increasingly used to detect defects in composites. However, IRT of composites faces more challenges due to their complex structure compared to that of monolithic-materials. In this article, the authors built model to perform IRT simulations of several types of defects found in composites. Subsequently IRT experiments were performed to verify simulation results. And then, Ultrasound C-scan, micro-CT and Terahertz NDT are preformed to conduct a comparative study of different NDT techniques. Finally, a comparison of the detection capabilities of different NDT techniques was conducted through matching and fusing of the results of experiments and simulations.
\end{abstract}

\section{Introduction}

This work provides researchers with appropriate verdicts to capitalize on the advantages of different NDT techniques to perform NDE on composites.

\section{Experiments and analysis}

\subsection{Ultrasound C-scan}

It is a challenge to detect thick 3-D CFRP using ultrasonic c-scan due to the CFRP complex internal structure. The complex internal structure can cause attenuation and scattering of ultrasonic beams. Ultrasonic c-scan has been widely used to detect voids and laminates in composites.

\subsection{Terahertz NDT}

Pulsed THz imaging is being adopted for NDT applications in military, aerospace and industrial settings, including for the analysis of pipes, radar domes and art. A key capability of this type of imaging is that it can be used to generate 2- or 3D subsurface images of many materials that are otherwise opaque.

In NDT, THz pulses are directed towards the sample as transmitter and receiver are 'raster scanned' in a 2D grid. At each point in the grid, the electric field of the pulse is recorded as a function of time. Imaging is performed either in transmission or reflection modes, providing different but complementary information.

\subsection{Micro-CT}

Application of micro-CT to composite materials was concentrated on metal-matrix and ceramic-matrix composites in the past. The spatial scale of features in these materials including fiber location and waviness, fiber breakage, local porosity and density, void volume, fatigue crack growth, etc. are accessible to micro-CT. However, recently some studies on polymer matrix composites have also been reported. A micro-CT inspection was performed. The resolution of the inspection is $18 \mu \mathrm{m}$. The purpose of the $x$-ray tomography inspection is to validate the infrared results.

\subsection{Infrared thermography}


Infrared thermography is increasingly used to detect composites. In the past few years, a great number of new infrared thermography methods have been proposed, such as eddy-current thermography, induction thermography, vibrothermography and laser excited thermography. Laser spot thermography (LST) has been used to detect surface cracks and fiber orientation in composites. Laser line thermography (LLT) has been used to detect surface cracks. However, the detection of other types of flaws was poorly documented. Li et al. used a beam expander and a cylindrical lens to convert a laser spot with a radius of around $0.9 \mathrm{~mm}$ to a laser line source. In this paper, an extremely fast swinging mirror is used to generate a laser line. A micro-lens was used to identify and characterize micro-sized flaws.

\section{Simulation and analysis}

A finite element simulation was performed to analyze the micro-porosities. A model was implemented into COMSOL Multiphysics as a user-defined material model for predicting the non-linear behavior of heat transmission in the sample. The model was validated for the experimental results. The geometrical model needed for finite element discretization was developed from the micro-CT measurements. The laser-line power is $2.9 \mathrm{~W}$. The power was tested in the lab. The power density is $2.9 \mathrm{~W} /(3 \mathrm{~mm} \times 10 \mathrm{~mm})$. The laser line covered an area of $2.5 \mathrm{~mm} \times 0.5 \mathrm{~mm}$. Therefore, the power in the model is $2.9 \mathrm{~W} \times(2.5 \mathrm{~mm} \times 0.5 \mathrm{~mm}) /(3 \mathrm{~mm} \times 10 \mathrm{~mm})$. The heating time is $0.5 \mathrm{~s}$.

\section{Conclusion}

This paper reveals the detection probability of different NDT techniques on composites.

\section{a. Figures}

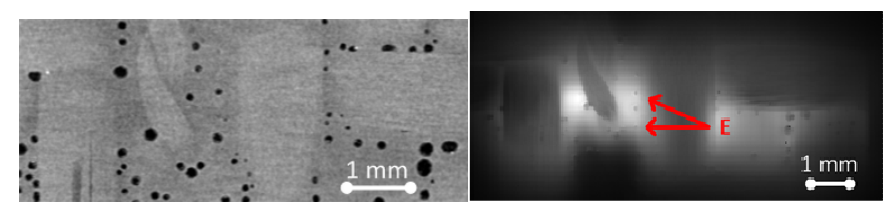

(a)

(d)

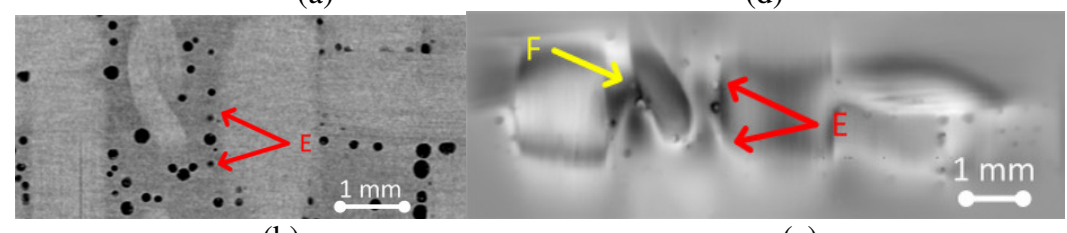

(b)

(e)

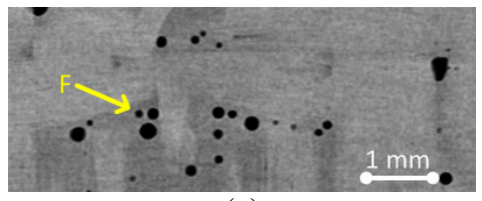

(c)

Figure 1: The comparison of micro-CT and micro-LLT results from the position of $129 \mathrm{~mm}$ : (a) the micro-CT slice on surface, (b) the micro-CT slice from the depth of $36 \mu \mathrm{m}$, (c) the micro-CT slice from the depth of $0.162 \mathrm{~mm}$, (d) the micro-LLT result from cold image subtraction, (e) the micro-LLT result from PCT.

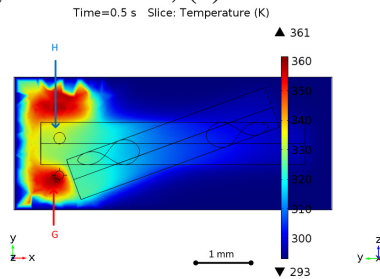

(a)

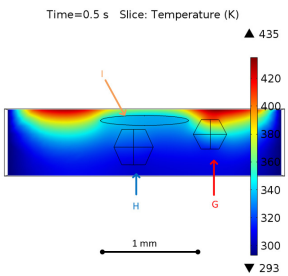

(b)

Figure 2: Slice temperature distribution when the heating time is $0.5 \mathrm{~s}$ : (a) top view, (b) side view.

\section{REFERENCES}

[1] H. Zhang, U. Hassler, M. Genest, H. Fernandes, F. Robitaille, et al., "Comparative study on submillimeter flaws in stitched T-joint carbon fiber reinforced polymer by infrared thermography, microcomputed tomography, ultrasonic c-scan and microscopic inspection," Opt. Eng. 54(10), 104109 (2015). 